\title{
JPSS-1 VIIRS version 2 at-launch relative spectral response characterization and performance
}

\author{
Chris Moeller $^{\mathrm{a} 1}$, Tom Schwarting ${ }^{\mathrm{b}}$, Jeff McIntire ${ }^{\mathrm{b}}$, Dave Moyer ${ }^{\mathrm{c}}$, and Jinan Zeng ${ }^{\mathrm{d}}$ \\ ${ }^{a}$ CIMSS, Univ. Wisconsin; 1225 West Dayton Street; Madison, WI \\ ${ }^{\mathrm{b}}$ Science Systems and Applications Inc., Lanham, MD \\ ${ }^{\mathrm{c}}$ The Aerospace Corporation, El Segundo, CA \\ ${ }^{\mathrm{d}}$ Fibertek Corporation, Herndon, VA
}

\begin{abstract}
The relative spectral response (RSR) characterization of the JPSS-1 VIIRS spectral bands has achieved "atlaunch" status in the VIIRS Data Analysis Working Group February 2016 Version 2 RSR release. The Version 2 release improves upon the June 2015 Version 1 release by including December 2014 NIST TSIRCUS spectral measurements of VIIRS VisNIR bands in the analysis plus correcting CO2 influence on the band M13 RSR. The T-SIRCUS based characterization is merged with the summer 2014 SpMA based characterization of VisNIR bands (Version 1 release) to yield a "fused" RSR for these bands, combining the strengths of the T-SIRCUS and the SpMA measurement systems. The M13 RSR is updated by applying a model-based correction to mitigate $\mathrm{CO} 2$ attenuation of the SpMA source signal that occurred during M13 spectral measurements. The Version 2 release carries forward the Version 1 RSR for those bands that were not updated (M8-M12, M14-M16A/B, I3-I5, DNBMGS). The Version 2 release includes band average (over all detectors and subsamples) RSR plus supporting RSR for each detector and subsample. The at-launch band average RSR have been used to populate Look-Up Tables supporting the sensor data record and environmental data record at-launch science products. Spectral performance metrics show that JPSS-1 VIIRS RSR are compliant on specifications with a few minor exceptions. The Version 2 release, which replaces the Version 1 release, is currently available on the password-protected NASA JPSS-1 eRooms under EAR99 control.
\end{abstract}

Keywords: JPSS-1, VIIRS, RSR, SDR

\section{INTRODUCTION}

The Joint Polar Satellite System-1 (JPSS-1, henceforth referred to as "J1" in this paper) Visible Infrared Imaging Radiometer Suite (VIIRS) instrument ${ }^{[1]}$, the second in the series (S-NPP VIIRS launched in 2011), is approaching an anticipated launch in the 1st quarter of 2017. Accordingly, pre-launch performance characterization has been completed and Look-Up Tables supporting the sensor data record (SDR) and environmental data record (EDR) algorithms have been generated. This includes the designation of "atlaunch" relative spectral response (RSR) in the form of the VIIRS Data Analysis Working Group (DAWG) February 2016 Version 2 (V2) RSR release. The V2 RSR release improves upon and replaces the June 2015 Version $1(\mathrm{~V} 1)$ release $^{[2]}$.

\footnotetext{
${ }^{1}$ Chris.Moeller@ssec.wisc.edu, 608-263-7494, 608-262-5974 (FAX), jeffrey.mcintire@ssaihq.com,
} thomas.schwarting@ssaihq.com, david.i.moyer@aero.org.jinan.zeng@nasa.gov 
Table 1. J1 VIIRS specifications and measured spectral performance of the DAWG V2 band average RSR, plus S-NPP at-launch integrated out-of-band (IOOB) performance. Red boxes indicate specification exceeded.

Focal Plane Legend: $\square$ - VisNIR; $\square$-S/MWIR; $\square$ - LWIR

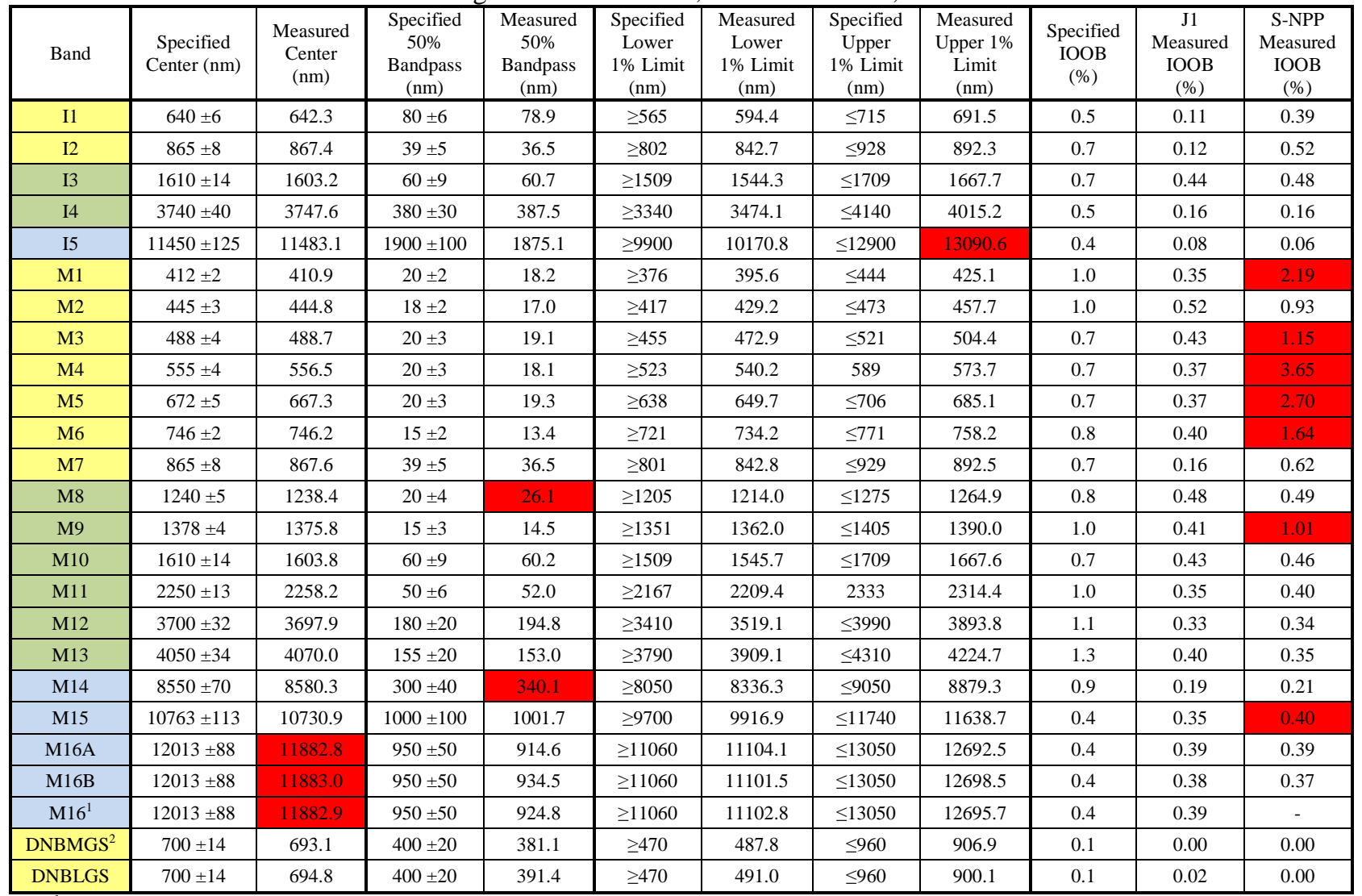

${ }^{1} \mathrm{M} 16$ is an average of M16A and M16B.

${ }^{1}$ DNBMGS spectral characterization represents DNBHGS. DNBHGS not directly measured due to its high gain.

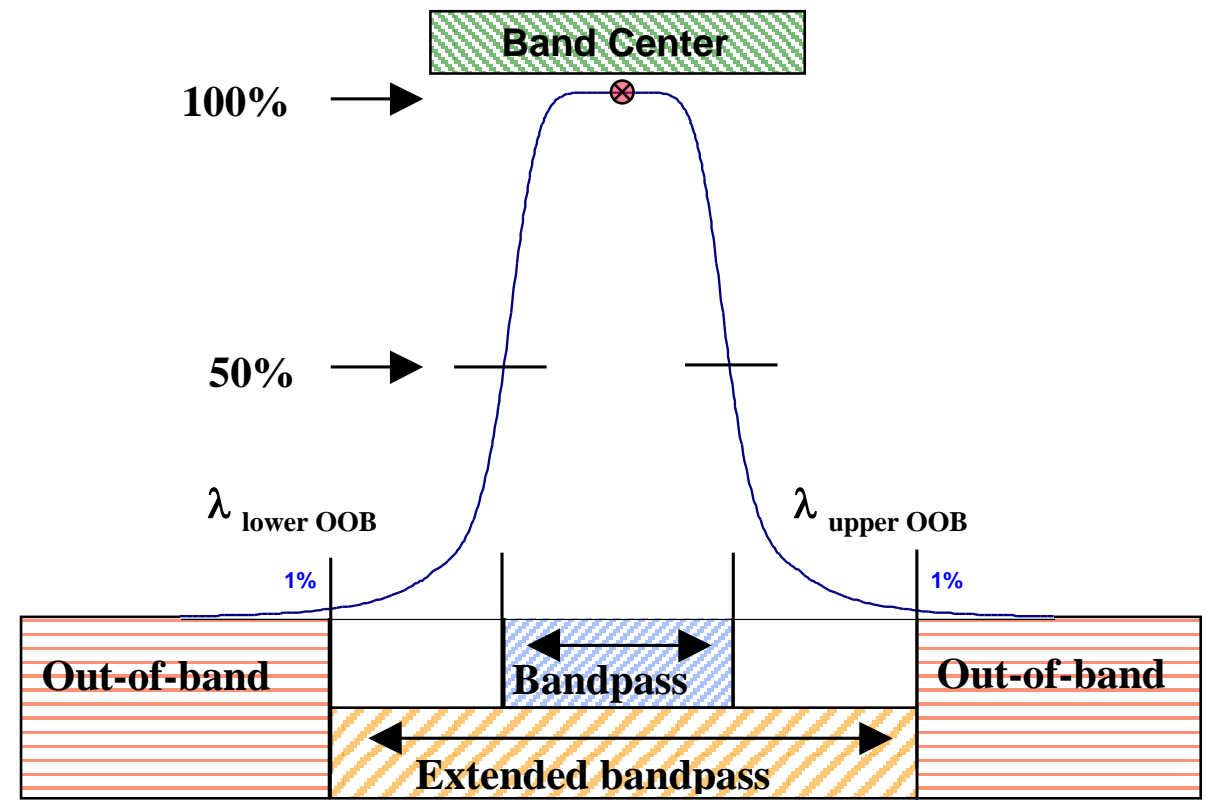

Figure 1. Graphical representation of VIIRS spectral performance specification metrics. 


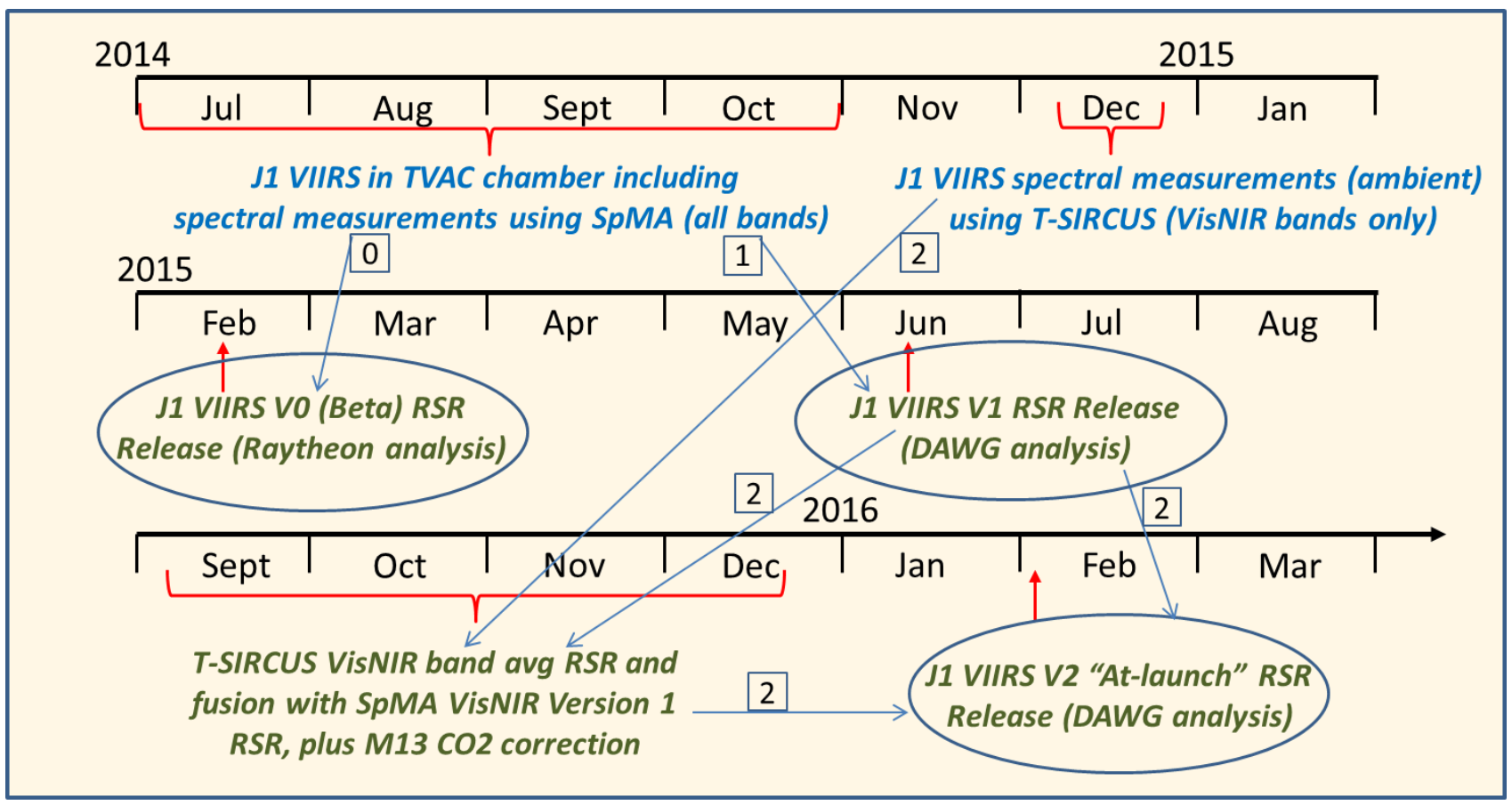

Figure 2. Pedigree of the three J1 VIIRS RSR releases (circled). Arrows with boxed numbers representing Version number connect measurements to releases. Both the February 2015 V0 and June 2015 V1 releases were based upon analysis of the summer/fall 2014 SpMA based spectral measurements. The V2 release incorporates the December 2014 NIST T-SIRCUS measurements into the analysis for VisNIR bands.

The government-sponsored VIIRS DAWG was tasked to generate the at-launch VIIRS RSR for J1. The RSR describe the in-band and out-of-band spectral dependence (Figure 1) of the light reaching the VIIRS detectors for each of the VIIRS $750 \mathrm{~m}$ resolution Moderate "M" bands and $375 \mathrm{~m}$ resolution Imaging "I" bands plus low and mid gain stages of the $750 \mathrm{~m}$ resolution Day-Night "DNB" band (Table 1). The DAWG, consisting of subject matter experts (SMEs) from the NASA VIIRS Characterization Support Team (VCST), Aerospace Corporation, and the University of Wisconsin, participated on-site during the J1 VIIRS spectral measurements at the Raytheon El Segundo facility in summer/fall 2014 and again in December 2014, gaining insight on the measurement data and instrument performance as well as providing input to Raytheon on path forward decisions. The analysis of the measurements leading to the V2 RSR release leveraged experience gained in a similar effort to analyze the S-NPP VIIRS RSR measurements ${ }^{[3,4]}$. In addition, the V2 RSR were evaluated on four performance metrics (Figure 1): band center (center wavelength between 50\% response levels), bandpass at 50\% response, extended bandpass at $1 \%$ response (a.k.a. in-band response) and the maximum integrated out-of-band response (ratio of integrated out-of-band response to integrated in-band response). This paper describes the development and performance of the J1 VIIRS V2 RSR.

\section{BACKGROUND ON MEASUREMENTS AND RSR VERSIONS}

Figure 2 provides a schematic linking the J1 VIIRS spectral measurements to the various releases of J1 VIIRS RSR. Both the V0 (beta) and V1 releases were based entirely upon the Spectral Measurement Assembly $\left(\right.$ SpMA $^{[5]}$ ) spectral measurements of all VIIRS bands in the summer/fall of 2014 at the Raytheon El Segundo facility. The SpMA is a dual monochromator system using tungsten bulb and ceramic glow bar sources. For the measurements, a slit reticle was placed in the beam path so that the illumination by the monochromatic light exiting the SpMA was constrained to a single band on the VIIRS focal plane (Figure 3) as the SpMA 


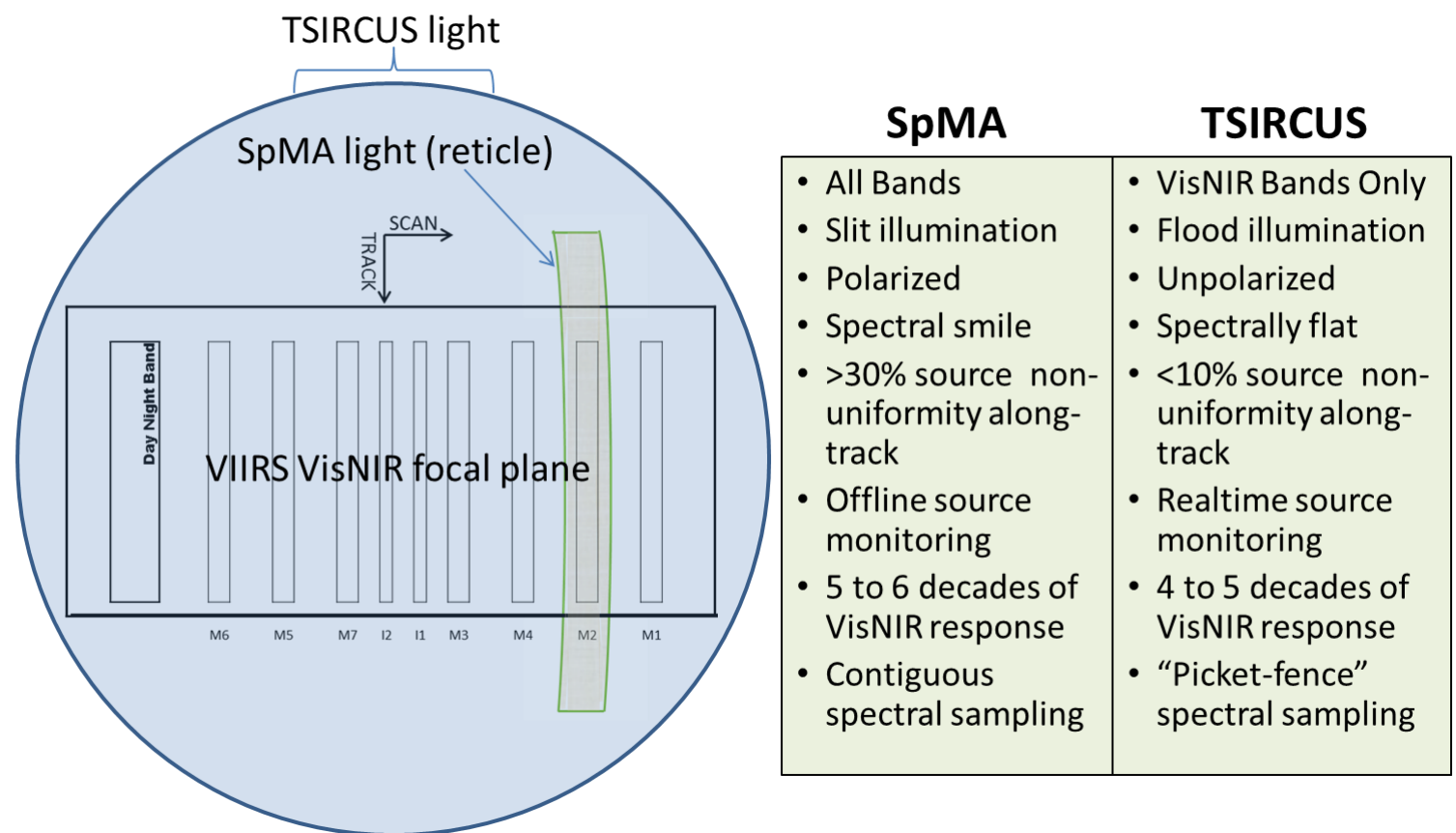

Figure 3. VIIRS VisNIR focal plane illumination (left) during the summer-fall 2014 spectral measurements using the SpMA (example of band M2 illumination) and during the December 2014 spectral measurements using TSIRCUS (entire focal plane illuminated). Characteristics of each measurement system are given at right.

was stepped through a range of wavelengths. This system level measurement highlights the performance of the filters overlying the detectors of each focal plane. The SpMA measurement setup contrasts with that employed in December 2014 for the T-SIRCUS (Traveling Spectral Irradiance and Radiance Responsivity with Uniform Sources) facility ${ }^{[6,7]}$ developed and maintained by NIST (National Institute of Standards and Technology). At the Raytheon facility, the T-SIRCUS laser-based source was coupled with fiber optics to transfer signal to an integrating sphere aligned to fully illuminate the VIIRS entrance aperture. This provided a flight-like flood illumination of the entire VIIRS VisNIR focal plane (Figure 3). Flood illumination results in an integrated response from VIIRS that includes elements of the filter performance plus optical and electronic effects, i.e. crosstalk, that may be present on the focal plane. Due to constraints of the J1 VIIRS cryoradiator and the ambient test environment, spectral measurements using T-SIRCUS were limited to the VIIRS VisNIR focal plane bands. These measurements, analyzed by the DAWG in September - December 2015, and a CO2 correction to M13 are the new elements of the DAWG February 2016 V2 RSR release.

The V0 (beta) release includes only detector RSR that were generated by Raytheon Corp analysts with DAWG SME oversight and input. Detector and band average (over all detectors and subsamples) RSR for the $\mathrm{V} 1$ release, which replaced the $\mathrm{V} 0$ release, were generated in an independent analysis by the DAWG ${ }^{[2]}$ and were used in early testing of VIIRS science algorithms. For V2, which also includes detector and band average RSR, the additional VisNIR band measurements using T-SIRCUS were analyzed by the DAWG and then merged with the V1 RSR for an updated "fused" RSR for these bands. The V2 release also includes an update of one thermal emissive band, M13, which was corrected to remove ambient $\mathrm{CO} 2$ influence on the source signal during the SpMA measurements. For those bands that were not updated for V2 (M8-M12, M14-M16A/B, I3-I5, DNBMGS), the V1 detector and band average RSR were carried forward into V2 to provide a comprehensive set of RSR in the V2 release, allowing the V2 release to replace the V1 release. Details of the analysis leading to the V2 RSR release are provided in the next section. 


\section{T-SIRCUS SPECTRAL MEASUREMENTS AND THE DAWG RSR ANALYSIS}

In the support of engineering test procedure 430 (ETP-430), the NIST T-SIRCUS system was deployed to the Raytheon El Segundo facility in the fall of 2014 and setup in an anteroom with an objective of measuring J1 VIIRS spectral response and polarization performance for VisNIR bands. T-SIRCUS was used previously to measure S-NPP VIIRS VisNIR band RSR at Ball Aerospace in Spring 2010 ${ }^{[4]}$. The VIIRS instrument was set up in an adjacent ambient clean room environment at Raytheon El Segundo with the $25 \mathrm{~cm}$ exit aperture of a $100 \mathrm{~cm}$ spectralon-coated integrating sphere aligned to the VIIRS rotating telescope assembly (RTA), with no intervening optical elements in the $\sim 0.9 \mathrm{~m}$ path between the sphere exit aperture and the VIIRS entrance aperture. The RTA was fixed (not rotating) during all spectral measurements. Fiber optic cables delivered the signal from the curtained T-SIRCUS laser system to the sphere. To cover the $380-1000 \mathrm{~nm}$ spectral range of the VisNIR in-band and out-of-band response measurements, T-SIRCUS used multiple tunable lasers including Ti:Sapphire ( 670-1000 nm), Dye R6G $(\sim 560-610 \mathrm{~nm})$, Dye DCM $(\sim 600-680 \mathrm{~nm})$, and doubled LBO OPO ( $\sim 380-565 \mathrm{~nm})$. T-SIRCUS produces nearly monochromatic light with a bandpass ranging from $0.001 \mathrm{~nm}$ to about $0.1 \mathrm{~nm}$ depending on the laser in use. Co-incident with the spectral measurements, the output of the sphere was sampled nearly continuously by a radiance monitor mounted on the sphere and viewing the interior wall opposite of the sphere aperture. The monitor data were used to produce an average sphere radiance and standard deviation for each measured wavelength. The sphere radiance monitor was calibrated twice using the NIST LTD-107 radiometer, once before the onset of the spectral measurement program and updated after the completion of the measurement program.

During the measurements, the T-SIRCUS laser in use was stepped through a series of wavelengths (a "sweep"), using a sequence consisting of a nominal 8, 21, or 28 second dwell or "collect" with a shutter blocking the source light ("shutter closed") to capture the dark background signal, followed by a similarly timed collect with the source light on the detectors ("shutter open"). The longer collects improved the signalto-noise ratio (SNR) in low signal spectral regions. During a typical 21 second collect, VIIRS recorded 2048 samples in each of about 11 "scans" (nominal 1.7864 seconds/scan) resulting in $>20000$ samples per collect ( $\sim 8000$ and 30000 samples, resp. for 8 and 28 second collects). Since the RTA was not rotating, all data were collected on one side of the dual-sided Half Angle Mirror. After completing the shutter open collect, the shutter was closed and the laser wavelength was tuned to the next setting until the sweep was completed or the laser mode had reached its useful spectral limit. Near the limits of adjacent laser modes, overlapping spectral collects were obtained to help reveal any biases between laser modes in the transition regions. Table 2 summarizes elements of the data collects. Overlapping in-band and out-of-band sweeps were necessary to avoid digital saturation by the powerful Ti:Sapphire laser in the high response zone of bands M6 and M7 (sweeps 2-7, 10-13); saturated data was discarded. It is also noteworthy that the laser bandwidths are significantly smaller than the wavelength step. This "picket fence" sampling approach using T-SIRCUS was evaluated during S-NPP VIIRS pre-launch testing and found to successfully capture the spectral response shape and amplitude when compared to the contiguous spectral measurements collected using the SpMA ${ }^{[4]}$.

To eliminate noisy measurements due to T-SIRCUS laser source instability, shutter open collects for which the sphere radiance standard deviation (as measured by the radiance monitor) exceeded $2 \%$ (of signal) were discarded. Shutter closed collects were averaged and subtracted from remaining averaged shutter open collects to provide a background corrected signal ("dn") at each wavelength $\lambda$

$$
d n(\lambda)=\overline{D N(\lambda)}_{\text {open }}-\overline{D N(\lambda)}_{\text {closed }} .
$$

Samples from scans at the beginning and end of the collects were generally discarded to avoid any partial scans (i.e. change of shutter status during the scan) being included in the averaging process. A standard deviation statistic for DN was also computed for each scan for assigning data quality (described in step 2 
Table 2. J1 VIIRS spectral test data collection characteristics using T-SIRCUS.

\begin{tabular}{|c|c|c|c|c|c|c|c|c|}
\hline $\begin{array}{c}\text { Sweep } \\
\text { number }\end{array}$ & Target & Date & Laser Mode & $\begin{array}{c}\text { Nominal } \\
\text { Laser } \\
\text { Bandwidth } \\
(\mathrm{nm})\end{array}$ & $\begin{array}{c}\text { Spectral } \\
\text { Stepsize } \\
(\mathrm{nm})\end{array}$ & $\begin{array}{l}\text { Light } \\
\text { Time } \\
\text { (sec) }\end{array}$ & $\begin{array}{l}\text { Dark } \\
\text { Time } \\
\text { (sec) }\end{array}$ & Description \\
\hline 1 & M7 IB & $12 / 5 / 2014$ & Ti:Sapphire & 0.001 & 1.0 & 5 & 3 & 835-901 nm Dry Run \\
\hline 2 & M7 IB & $12 / 5 / 2014$ & Ti:Sapphire & 0.001 & 1.0 & 8 & 8 & 835-901 nm First run \\
\hline 3 & M7 IB & $12 / 5 / 2014$ & Ti:Sapphire & 0.001 & 1.0 & 8 & 8 & 835-901 nm Interleave \\
\hline 4 & M7 IB & $12 / 5 / 2014$ & Ti:Sapphire & 0.001 & 1.0 & 8 & 8 & $835-901 \mathrm{~nm}$ Interleave \\
\hline 5 & M7 IB & $12 / 5 / 2014$ & Ti:Sapphire & 0.001 & 1.0 & 8 & 8 & $835-901 \mathrm{~nm}$ Interleave \\
\hline 6 & M6 IB & $12 / 5 / 2014$ & Ti:Sapphire & 0.001 & 0.4 & 8 & 8 & 729-765 nm First run \\
\hline 7 & M6 IB & $12 / 5 / 2014$ & Ti:Sapphire & 0.001 & 0.4 & 8 & 8 & $729-765 \mathrm{~nm}$ Interleave \\
\hline 8 & M5 IB & $12 / 6 / 2014$ & Dye DCM & 0.01 & 0.2 & 8 & 8 & $640-668 \mathrm{~nm}$ \\
\hline 9 & M5 IB & $12 / 6 / 2014$ & Ti:Sapphire & 0.001 & 0.2 & 8 & 8 & $667-680 \mathrm{~nm}$ \\
\hline 10 & OOB1 & $12 / 6 / 2014$ & Ti:Sapphire & 0.001 & 1.0 & 21 & 21 & $800-899 \mathrm{~nm}$ \\
\hline 11 & OOB2 & $12 / 6 / 2014$ & Ti:Sapphire & 0.001 & 1.0 & 21 & 21 & $700-800 \mathrm{~nm}$ \\
\hline 12 & OOB3 & $12 / 6 / 2014$ & Ti:Sapphire & 0.001 & 1.0 & 21 & 21 & $899-1001 \mathrm{~nm}$ \\
\hline 13 & OOB4 & $12 / 7 / 2014$ & Ti:Sapphire & 0.001 & 0.2 & 21 & 21 & $680-703 \mathrm{~nm}$ \\
\hline 14 & OOB5 & $12 / 8 / 2014$ & Dye DCM & 0.01 & 1.0 & 21 & 21 & $630-685 \mathrm{~nm}$ \\
\hline 15 & M5 IB & $12 / 8 / 2014$ & Dye DCM & 0.01 & 0.2 & 21 & 21 & $595-660 \mathrm{~nm}$ \\
\hline 16 & OOB6 & $12 / 8 / 2014$ & Dye DCM & 0.01 & 1.0 & 21 & 21 & 631-606 nm \\
\hline 17 & OOB7 & $12 / 8 / 2014$ & Dye R6G & 0.01 & 0.2 & 21 & 21 & $562-582 \mathrm{~nm}$ \\
\hline 18 & OOB8 & $12 / 8 / 2014$ & Dye R6G & 0.01 & 1.0 & 21 & 21 & $578-606 \mathrm{~nm}$ \\
\hline 19 & M1 IB & $12 / 9 / 2014$ & LBO OPO & 0.1 & 0.5 & 28 & 28 & $417-430 \mathrm{~nm}$ \\
\hline 20 & M1 IB & $12 / 9 / 2014$ & LBO OPO & 0.1 & 0.5 & 28 & 28 & $391-417 \mathrm{~nm}$ \\
\hline 21 & M2 IB & $12 / 9 / 2014$ & LBO OPO & 0.1 & 0.5 & 28 & 28 & $422-463 \mathrm{~nm}$ \\
\hline 22 & M1 IB & $12 / 9 / 2014$ & LBO OPO & 0.1 & 0.25 & 28 & 28 & $417-431 \mathrm{~nm}$ \\
\hline 23 & M4 IB & $12 / 10 / 2014$ & LBO OPO & 0.1 & 0.5 & 28 & 28 & $535-548 \mathrm{~nm}$ \\
\hline 24 & M4 IB & $12 / 10 / 2014$ & LBO OPO & 0.1 & 0.5 & 28 & 28 & $545-558 \mathrm{~nm}$ \\
\hline 25 & M3 IB & $12 / 10 / 2014$ & LBO OPO & 0.1 & 0.5 & 28 & 28 & $463-509 \mathrm{~nm}$ \\
\hline 26 & M3 IB & $12 / 10 / 2014$ & LBO OPO & 0.1 & 0.5 & 28 & 28 & $466-487 \mathrm{~nm}$ \\
\hline 27 & M3 IB & $12 / 10 / 2014$ & LBO OPO & 0.1 & 0.5 & 28 & 28 & $505-512 \mathrm{~nm}$ \\
\hline 28 & OOB9 & $12 / 10 / 2014$ & LBO OPO & 0.1 & 1.0 & 28 & 28 & $510-535 \mathrm{~nm}$ \\
\hline 29 & M1 IB & $12 / 10 / 2014$ & LBO OPO & 0.1 & 0.5 & 28 & 28 & $391-395 \mathrm{~nm}$ \\
\hline 30 & M1 IB & $12 / 10 / 2014$ & LBO OPO & 0.1 & 0.5 & 28 & 28 & $391-417 \mathrm{~nm}$ \\
\hline 31 & OOB10 & $12 / 10 / 2014$ & LBO OPO & 0.1 & 1.0 & 28 & 28 & $379-390 \mathrm{~nm}$ \\
\hline 32 & M4 IB & $12 / 10 / 2014$ & LBO OPO & 0.1 & 0.5 & 28 & 28 & $539-563 \mathrm{~nm}$ \\
\hline 33 & OOB11 & $12 / 10 / 2014$ & LBO OPO & 0.1 & 2.0 & 28 & 28 & $490-560 \mathrm{~nm}$ \\
\hline 34 & OOB12 & $12 / 11 / 2014$ & LBO OPO & 0.1 & 2.0 & 28 & 28 & $380-420 \mathrm{~nm}$ \\
\hline 35 & OOB13 & $12 / 11 / 2014$ & LBO OPO & 0.1 & 2.0 & 28 & 28 & $419-470 \mathrm{~nm}$ \\
\hline 36 & OOB14 & $12 / 11 / 2014$ & Dye DCM & 0.01 & 1.0 & 21 & 21 & $634-685 \mathrm{~nm}$ \\
\hline 37 & OOB15 & $12 / 11 / 2014$ & Dye DCM & 0.01 & 2.0 & 21 & 21 & $604-631 \mathrm{~nm}$ \\
\hline 38 & OOB16 & $12 / 11 / 2014$ & Dye R6G & 0.01 & 2.0 & 21 & 21 & $562-635 \mathrm{~nm}$ \\
\hline 39 & M4 IB & $12 / 11 / 2014$ & LBO OPO & 0.1 & 0.5 & 28 & 28 & $555-562 \mathrm{~nm}$ \\
\hline 40 & M4 IB & $12 / 11 / 2014$ & LBO OPO & 0.1 & 0.5 & 28 & 28 & $555-563 \mathrm{~nm}$ \\
\hline
\end{tabular}

"IB" = in-band; "OOB" = out-of-band 
later in this section). The dn values for each detector were then calibrated to absolute spectral response (ASR) using the co-incident sphere radiance $\mathrm{L}_{\mathrm{Sph}}$ from the monitor observations

$$
\operatorname{ASR}(\lambda)=\frac{d n(\lambda)}{L_{S p h}(\lambda)} .
$$

Calibrating the response measurements to absolute response (i.e. ASR) allowed simple and effective combining of spectrally adjacent measurements from different sweeps without introducing biases due to source variation between sweeps. ASR was then converted to RSR by normalizing to the peak response

$$
R S R(\lambda)=\frac{A S R(\lambda)}{A S R_{\text {Max }}\left(\lambda_{A S R_{\text {Max }}}\right)}
$$

After generation of the T-SIRCUS based RSR, additional steps were taken leading to a high quality band average fused RSR in which the T-SIRCUS based RSR were merged with the SpMA based RSR from the June 2015 V1 release. The fused RSR combine the superior integrated response enabled by the flood illumination approach of the T-SIRCUS system in the high response zone, with the better quality data of the SpMA measurement system in the low response zone. The effect of these steps is graphically depicted in the example of Figure 4.

1. Remove inconsistent/rogue response data in the T-SIRCUS based RSR. This removes out-of-family response that has met the sphere radiance $2 \%$ standard deviation test but remains out-of-family. Compare upper left (baseline) to upper middle panel in Figure 4.

2. Apply a standard error of the mean (SEM) statistic threshold to the T-SIRCUS based RSR to distinguish high from low quality response. The SEM is the root sum square of the individual scan standard deviations for each wavelength divided by the square root of the sample size. The SEM of all detectors was averaged to a band average SEM. The band average SEM threshold was chosen subjectively for each band after close inspection of the response data for all detectors and is intended to separate noise-driven response from light-driven response. This is shown in the upper right panel.

3. Remove all low quality response from the T-SIRCUS based RSR. Shown in the lower left panel.

4. Merge high quality T-SIRCUS based RSR with SpMA based RSR to form a fused RSR. The spectral gaps left by discarding the low quality response in the T-SIRCUS based RSR are filled with SpMA based response. Any SpMA based response outside the spectral boundaries of the T-SIRCUS based response is also added. This is shown in the bottom middle panel.

5. Linearly average the fused RSR over all detectors and subsamples to make the band average RSR. Low quality response is assigned a response value of $1 \mathrm{E}-10$. This is depicted in the lower right panel. The band average fused RSR is provided in the V2 release of the J1 VIIRS VisNIR band RSRs (except DNBMGS which experienced saturation during T-SIRCUS based measurements) and is used to populate the SDR RSR Lookup Table (LUT) utilized by the J1 VIIRS SDR product algorithm.

In the M3 example of Figure 4, the noise floor is generally at or below 1E-04. This is typical of most VisNIR wavelengths for $\mathrm{M}$ bands, although the noise floor is typically slightly higher in the $470-520 \mathrm{~nm}$ region. The VisNIR M bands are all dual gain bands with the exception of M6 which is a single gain band but with a limited dynamic range. The VisNIR I bands however are single gain bands with gain settings intended to cover the full dynamic range of earth scenes; thus I band signal levels are much lower resulting in a noise floor that reaches and exceeds $1 \mathrm{E}-03$ over a portion of the measured wavelengths. For these bands, the majority of the out-of-band region is discarded as low quality response and thus the fusion with the SpMA measurements that support V1 adds considerable response information to the out-of-band region, assuring that all filter leaks down to $1 \mathrm{E}-05$ response or lower have been captured in the fused RSR. 

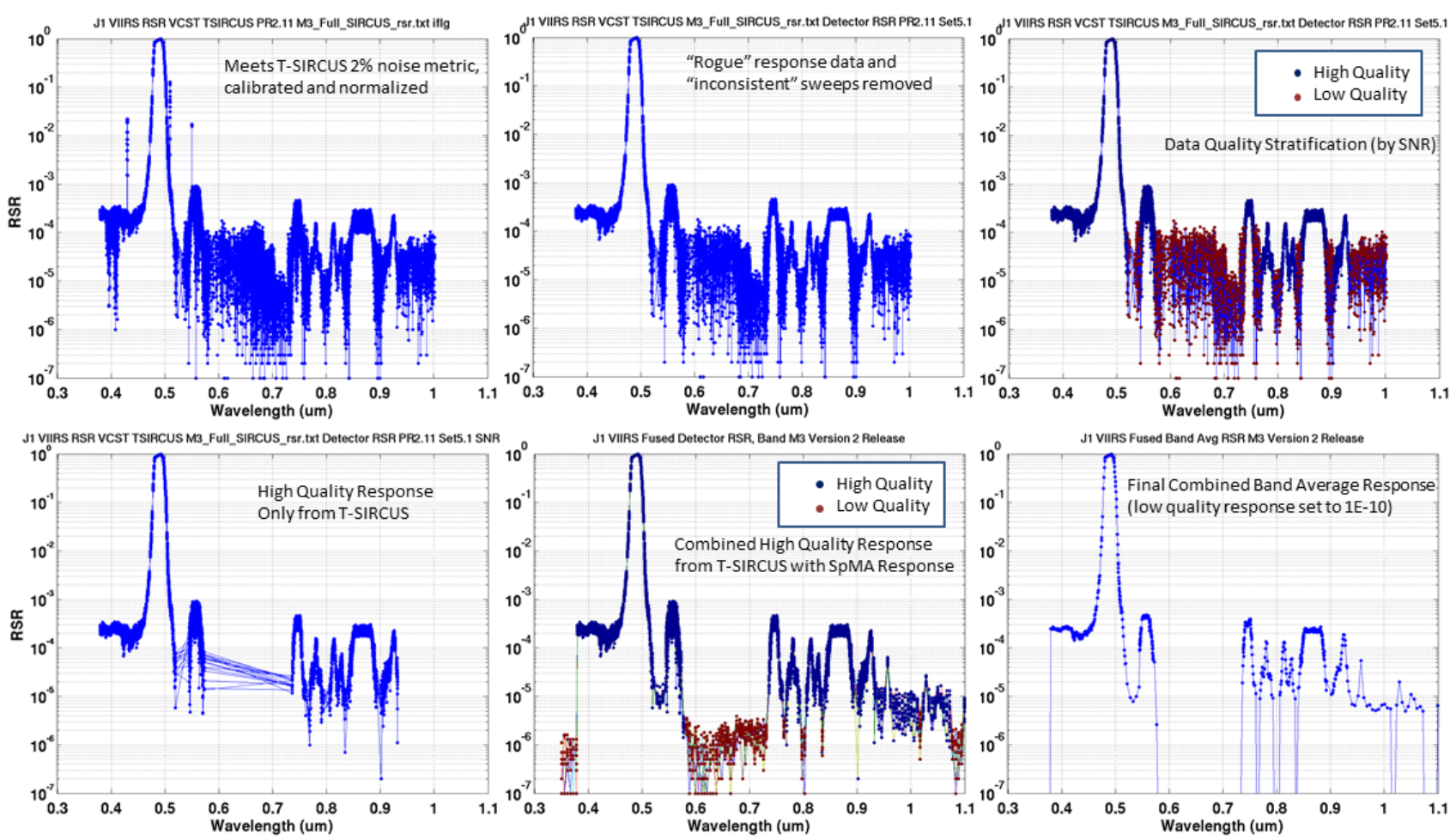

Figure 4. Band M3 sequential processing steps from detector RSR (upper left) to final band average RSR (lower right).

Band M13 was not measured during the T-SIRCUS based spectral measurements in December 2014, however the June 2015 M13 V1 RSR contains contamination from CO2 present during the SpMA based spectral measurements in the summer/fall 2014. A correction of that contamination was attempted for the V2 RSR release. M13 is positioned spectrally near a strong CO2 absorption feature, with overlap of the absorption feature on the red side low response wing of M13. The strength of the CO2 attenuation in the overlapping wavelengths coupled with the low response of the M13 RSR in that portion of the spectrum make it challenging to restore the true response level for those wavelengths, especially in light that the measurement program did not include real-time $\mathrm{CO} 2$ concentration monitoring. However, the LBLRTM radiative transfer model ${ }^{[8]}$ was applied in an attempt to estimate and correct the $\mathrm{CO} 2$ attenuation present during the M13 spectral measurements. Using a range of $\mathrm{CO} 2$ concentrations, model transmittances were compared to the M13 spectral measurements in an attempt to match the uncorrected observed shape of the M13 spectral measurements. From this effort a CO2 concentration of $700 \mathrm{ppm}$ was accepted for use in the model. A $3 \mathrm{~nm}$ spectral shift was also applied to the model calculation, improving the match (Figure 5, left panel). The measured relative spectral output (RSO) of the SpMA source was also corrected by discarding the RSO measurements contaminated by the $\mathrm{CO} 2$ attenuation and interpolating across the resulting spectral gap using the retained RSO measurement end points. The CO2 corrected M13 RSR was then given by

$$
\operatorname{RSR}(\lambda)_{M 13}=\frac{d n(\lambda)}{\tau(\lambda, T, C O 2)_{\text {ambient }} * \operatorname{RSO}(\lambda, T, C O 2)},
$$

where $\tau(\lambda, T, C O 2)_{\text {ambient }}$ represents the modeled atmospheric transmission over the $3.6 \mathrm{~m}$ ambient pathlength of the spectral measurement test setup for $\mathrm{M} 13$, and $\operatorname{RSO}(\lambda, T, C O 2)$ represents the $\mathrm{CO} 2$ corrected RSO. 

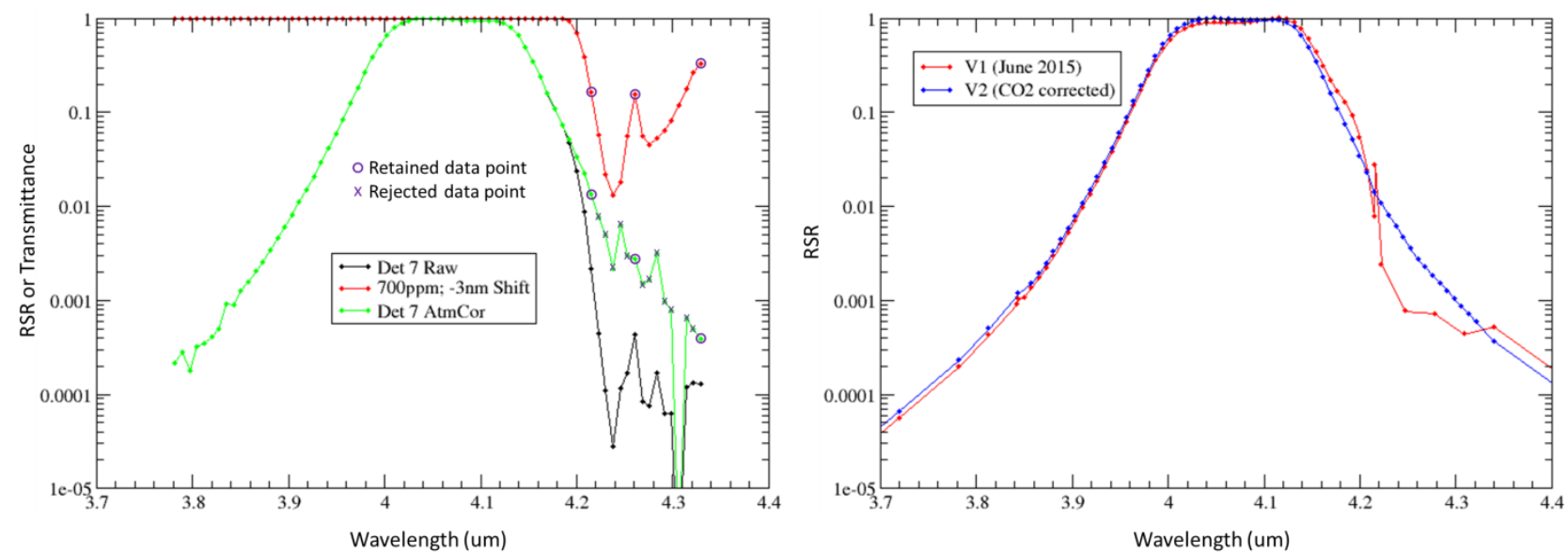

Figure 5. Band M13 raw response (black), model transmittance (red) and CO2 corrected response (green) for detector 7 example in left panel. Circled data points in the $\mathrm{CO} 2$ absorption region are retained as anchors for interpolating response at rejected wavelengths. Right panel shows final band average M13 RSR after CO2 correction and interpolation (blue). Improvement in the M13 RSR is self-evident when compared to the uncorrected V1 M13 RSR (red).

Of the $\mathrm{CO} 2$ corrected response data points, three wavelengths were retained as best representing the true response across the $\mathrm{CO} 2$ absorption region, largely due to their relatively high transmittance. These retained wavelengths (circled in Figure 5) were used as the tie points for a simple linear interpolation to replace the rejected wavelengths (" $\mathrm{x}$ " in Figure 5) in the $\mathrm{CO} 2$ absorption region. The final V2 band average M13 RSR is shown with the same for V1, revealing the improvement in the $\mathrm{CO} 2$ absorption region (Figure 5, right panel).

\section{DAWG VERSION 2 RSR RESULTS AND DISCUSSION}

The J1 VIIRS V2 RSR release consists of the updated fused RSR for VisNIR bands M1-M7, I1, I2, and DNBLGS and an updated RSR for band M13 with the balance of the bands (M8-M12, M14-M16A/B, I3-I5, DNBMGS) drawn from the J1 VIIRS V1 RSR release of June 2015. The comparison of V2 fused VisNIR RSR to V1 RSR shows modest shape change in the in-band region (Figure 6) and additional out-of-band response features (Figure 7). While there is little difference in spectral position and bandpass, the higher spectral density of the V2 T-SIRCUS based measurements provides a smoother shape in the in-band high response zone as well as a smoother shoulder transition to the lower response zone (Figure 6). The DNBLGS fused RSR in the high response zone is markedly changed with some broadening of the high response zone and a shift of energy blueward, likely due to the excellent spectral characterization of the source in the TSIRCUS measurements afforded by the real-time source monitoring.

In the out-of-band spectral region (Figure 7), additional response features caused by electronic crosstalk show up in V2 fused RSR. These features show up as reduced-amplitude manifestations of the RSR shape of the "sender" bands in the output of the "receiver" bands. The position of each M band is given by the vertical green lines in Figure 7 to help identify these features. For example, when $400 \mathrm{~nm}$ light is illuminating the focal plane, an in-band response is observed in band M1 output (i.e. in-band response caused by light passing through the M1 filter) as expected, but an unexpected out-of-band response is also observed in M2-M7 output (i.e. crosstalk response). The response in M2-M7 is understood to be a result of the illumination of band M1 with in-band light, which is distributed electronically (as opposed to optically) to bands M2-M7. There is no $400 \mathrm{~nm}$ response apparent in bands I1 and I2 in Figure 7; however electronic crosstalk does exist in these bands at the subsample level (I bands collect 2 subsamples for each $\mathrm{M}$ band sample). This electronic crosstalk from M1 essentially cancels out between the 2 subsamples of I1 and I2 in the band average RSR, i.e. 

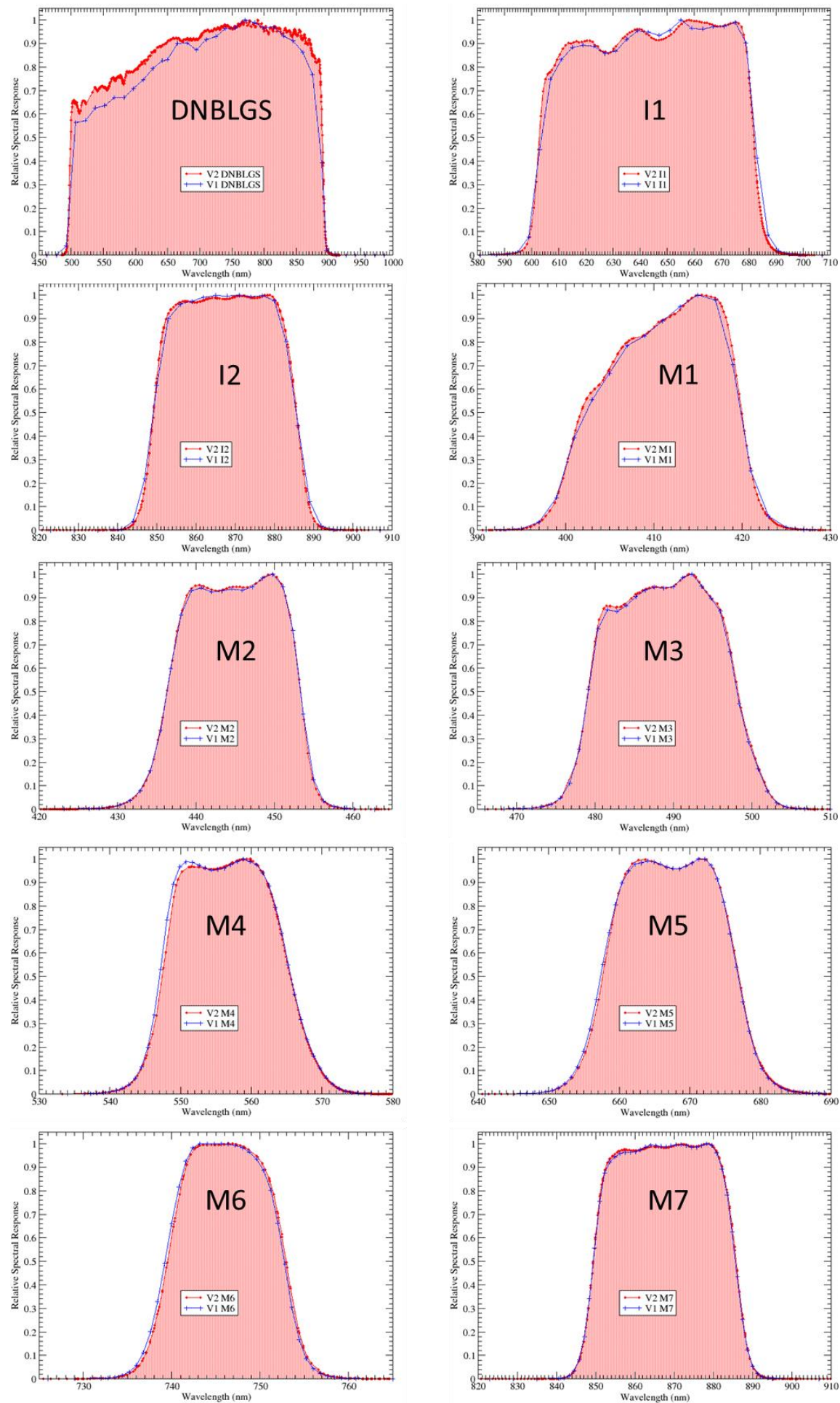

Figure 6. J1 band average fused RSR for V2 (red with shading) and V1 (blue) for the in-band spectral region. High density T-SIRCUS measurements for V2 resulted in improved definition of spectral shape, especially in the high response and "shoulders" of the V2 RSR. For the very broad DNBLGS RSR, which has high sensitivity to the source characterization, the T-SIRCUS source characterization has shifted energy to shorter wavelengths as compared to V1. 

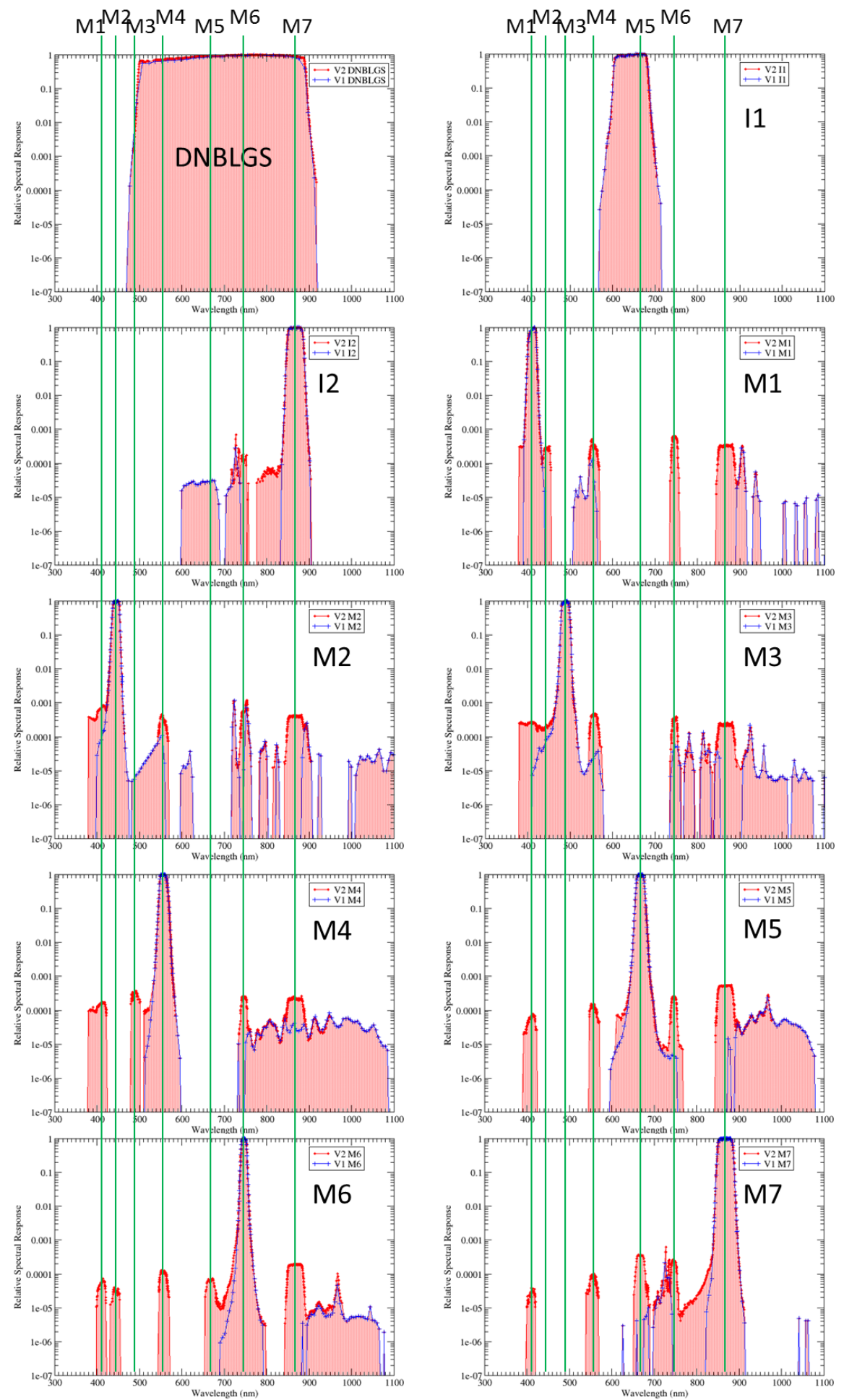

Figure 7. J1 band average RSR for V2 (red with shading) and V1 (blue) for the out-of-band spectral region. Electronic crosstalk features show up in many bands for V2. Green vertical lines represent band center positions of each band and are intended to help recognize sender band response in each receiver band, e.g. out-of-band response centered at about $870 \mathrm{~nm}$ (center of M7 in-band) is showing up in V2 RSR for M1-M6. 
the crosstalk from one subsample is positive and the crosstalk from the next subsample is roughly an equal negative. Nevertheless, the influence of electronic crosstalk will be present in unaggregated (i.e. single pixel) and in 3 pixel aggregated SDR data for J1 VIIRS I band observations (as it also is for S-NPP VIIRS). Out-of-band response exists at the spectral positions of all VisNIR M bands, though at varying signal levels for each receiver band. Fortunately, the amplitude of this electronic crosstalk driven response remains relatively small and within specification on out-of-band contributions to total signal; this will be further illustrated in the comparison of J1 V2 RSR to the at-launch S-NPP RSR that will follow later in this section.

There are many out-of-band features common to the V1 and V2 RSR. These features are associated with spectral leaks in the filters overlying the VisNIR band detectors. For example, local response peaks exist at about $780 \mathrm{~nm}$ and again at about $820 \mathrm{~nm}$ in band M3. This is out-of-band light that has passed through the M3 bandpass filter to reach the detector; the fact that the T-SIRCUS and SpMA measurements show nearly identical response at these wavelengths strongly suggests that both systems are accurately quantifying the response. Noticeable filter leaks exist in several bands, e.g. $900 \mathrm{~nm}$ in M1, $720 \mathrm{~nm}$ in M2, etc. Importantly, these spectral leaks are well characterized in the RSR so that their influence on the SDR and downstream EDR products can be quantified. Finally, there were no T-SIRCUS measurements beyond about $1000 \mathrm{~nm}$; V2 response information beyond $1000 \mathrm{~nm}$ was taken entirely from the SpMA measurements of V1.

The DAWG V2 band average RSR for J1 are graphically shown for all bands measured during the pre-launch test program in Figures 8 (in-band region) and 9 (out-of-band region). The October 2011 band average $\mathrm{RSR}^{[4]}$ for S-NPP are also included in the plots. Note that S-NPP reflective solar band RSR (M1-M11, I1-I3, DNBLGS, DNBMGS) have all undergone an on-orbit modulation caused by darkening of the rotating telescope assembly mirrors due to a tungsten contamination ${ }^{[9]}$; this darkening will not occur on J1 VIIRS and so for comparison purposes the S-NPP unmodulated October 2011 RSR are used here.

J1 VIIRS VisNIR RSR show spectral position shifts and shape changes in the high response zone compared to S-NPP (Figure 8). The VisNIR focal plane was redesigned for J1 to mitigate optical crosstalk caused by angle resolved scatter in the S-NPP VisNIR focal plane filters. While these RSR changes are noticeable, the J1 VIIRS VisNIR band average RSR fall within the center wavelength and bandpass performance metric requirements (see Table 1). Differences between S-NPP and J1 in-band RSR for S/MWIR, and LWIR bands are generally small and have been documented in a description of the J1 VIIRS V1 RSR ${ }^{[2]}$.

Differences in the S-NPP and J1 VIIRS out-of-band RSR are also noticeable for VisNIR bands (Figure 9). It is evident that bands I1, I2, and M1-M7 all show much less out-of-band response in the J1 RSR than in the SNPP RSR. The out-of-band response consists of filter leaks plus optical and electronic crosstalks. Optical crosstalk, which dominated much of the out-of-band response in S-NPP was greatly reduced with the J1 VisNIR focal plane redesign, leaving small filter leaks and electronic crosstalk as the remaining contributors to the J1 out-of-band response. Common electronic crosstalk features are identifiable in the S-NPP and J1 RSR, perhaps most easily recognized at the wavelengths of M7 in receiver bands M2 and M5, but present in all bands. All J1 VisNIR bands are compliant on the integrated out-of-band performance metric (Table 1). With exception of band M9, minor reductions or increases in out-of-band response are largely attributable to data quality differences at low response $(<1 \mathrm{E}-04)$ wavelengths. For S-NPP band M9, a response just outside of the in-band region contributed significantly to the integrated out-of-band response calculation. That feature is not present in $\mathrm{J} 1$, and may have been an artifact of the analysis for S-NPP.

While not presented in Figures 8 and 9, the DAWG J1 VIIRS V2 RSR also include an "M16" RSR, which is an average of the M16A and M16B RSR and is intended to represent response for an integrated signal of M16A and M16B. On-orbit nominal operations will downlink the integrated M16 signal for inclusion in the SDR product. The M16 RSR was drawn from the DAWG V1 RSR for J1. 

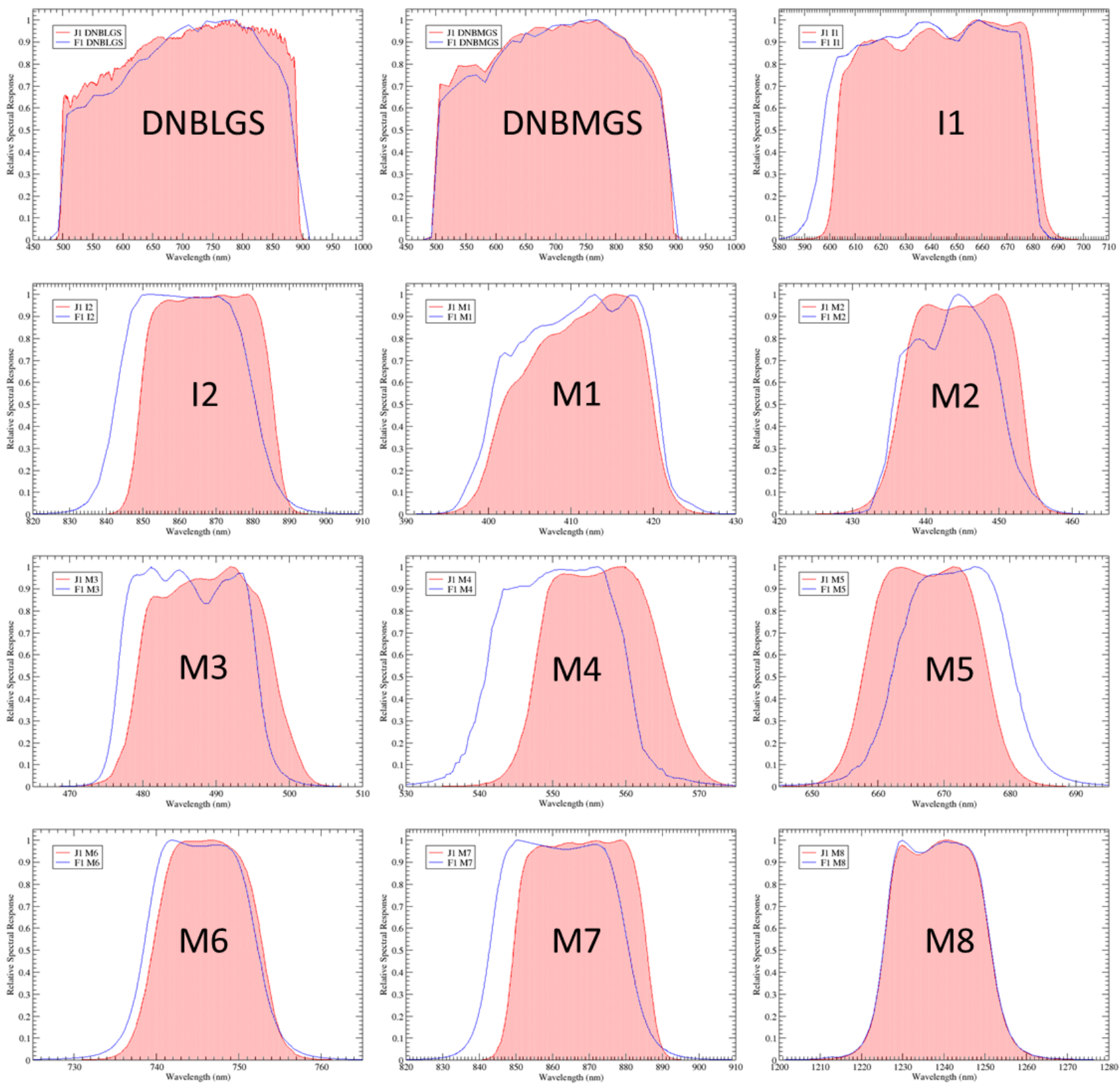

Figure 8. J1 (red shaded) and S-NPP (blue, labeled as "F1") band average RSR for the in-band spectral region. Spectral position and shape differences are prevalent for VisNIR bands (I1, I2, M1-M7) due to redesign of the J1 VIIRS VisNIR focal plane. SWIR band M8 (along with other SWIR bands shown in continuation of Figure 8) shows minor shape differences likely due to the redesign of J1 VIIRS dichroic \#2, which governs reflection and transmission of light to the S/MWIR and LWIR focal planes. 

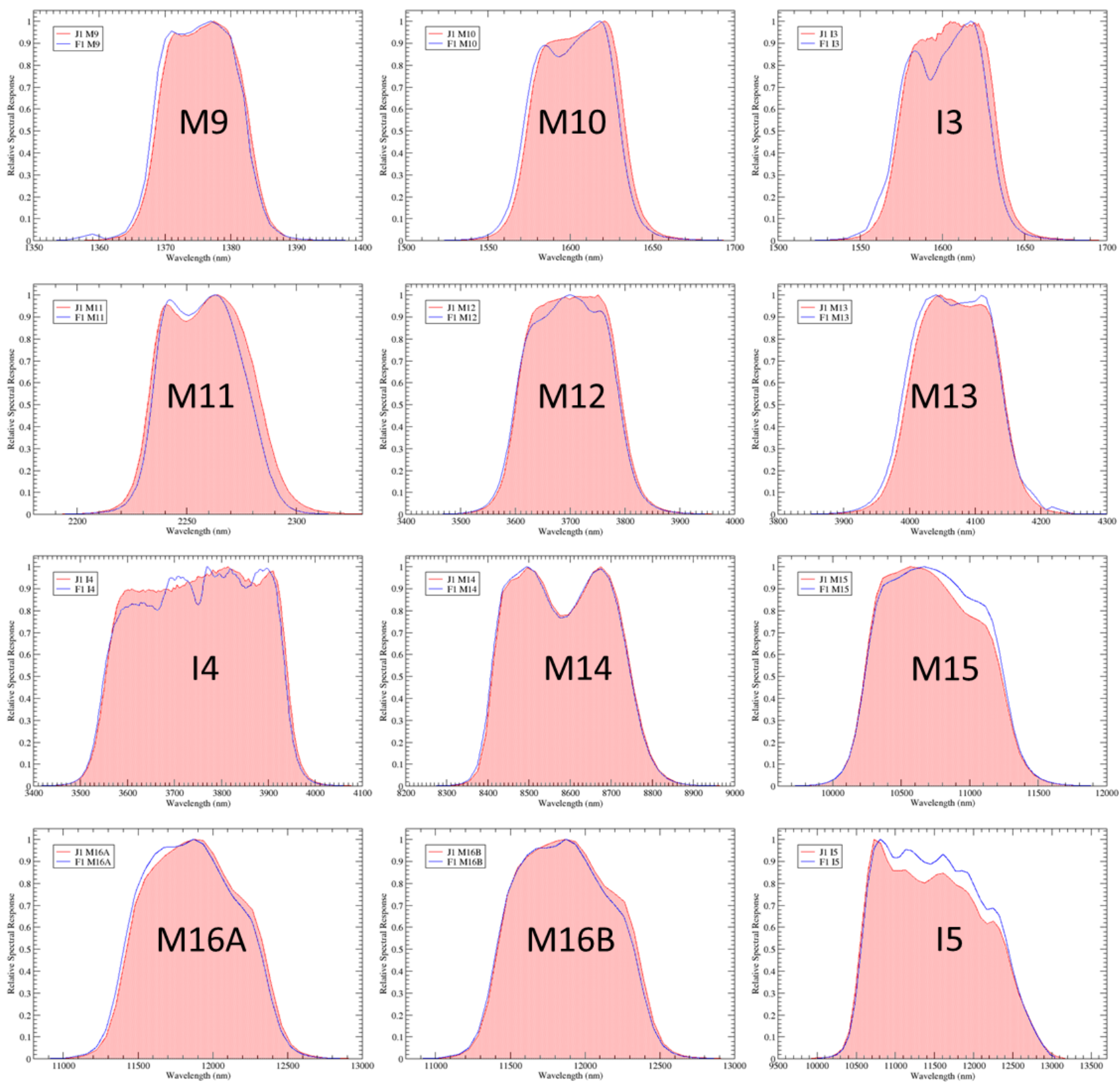

Figure 8 (Cont.). SWIR bands M9-M11, I3 and MWIR bands M12, M13, I4 all show minor shape differences between S-NPP and J1 while largely consistent on spectral position. LWIR bands M15, M16A/B and I5 also show minor shape differences while M14 remains largely unchanged. Shape differences likely due to redesign of J1 VIIRS dichroic \#2, as well as the correction to remove the influence of CO2 on the M13 RSR. 

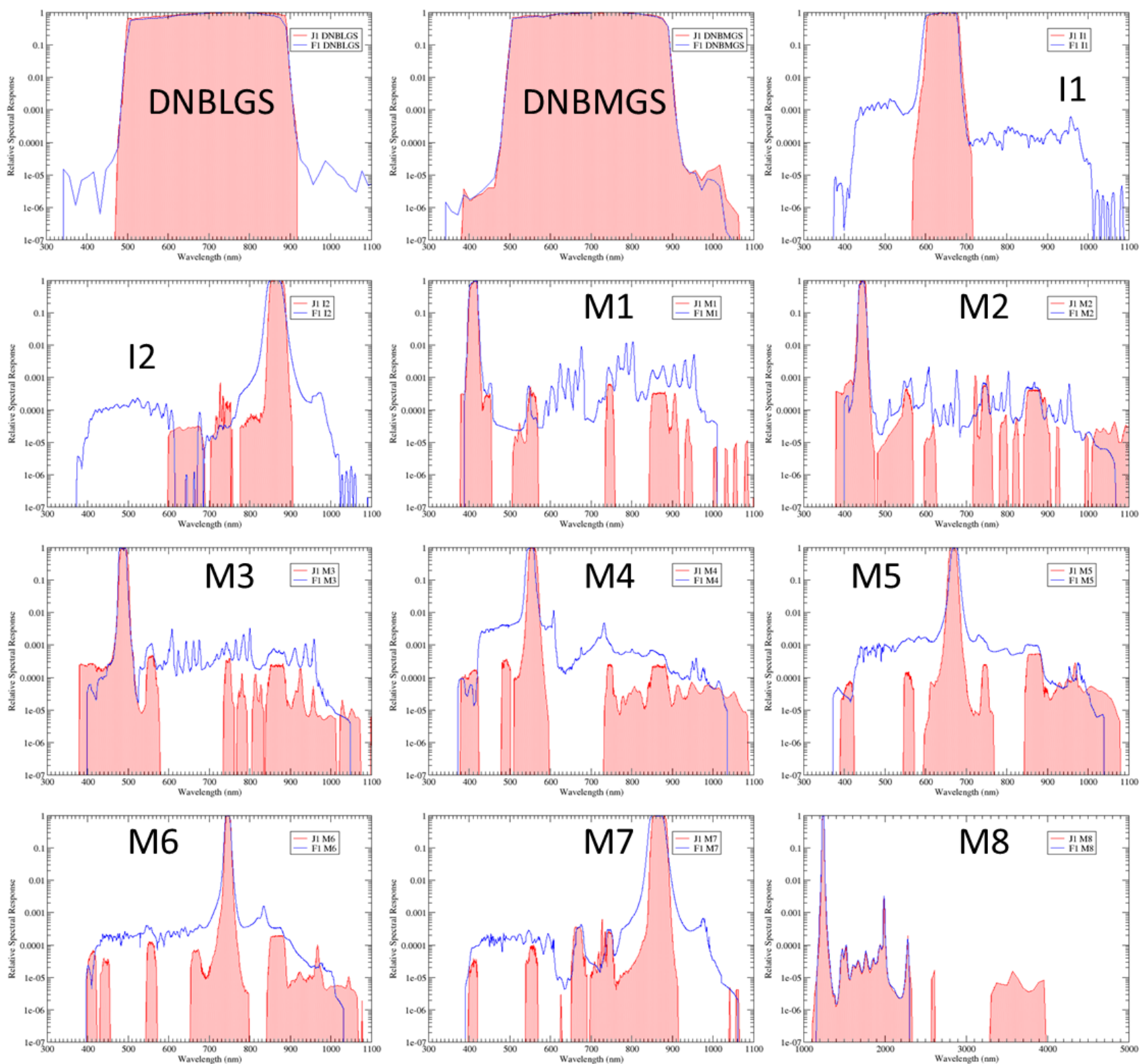

Figure 9. J1 (red shaded) and S-NPP (blue, labeled as "F1") band average RSR for the out-of-band spectral region. J1 VIIRS out-of-band response is significantly reduced for all J1 VisNIR bands compared to S-NPP due to the redesign of the J1 VIIRS VisNIR focal plane, bringing the integrated out-of-band performance metric into compliance for the J1 bands. J1 SWIR band M8 out-of-band performance is comparable to that of S-NPP. 

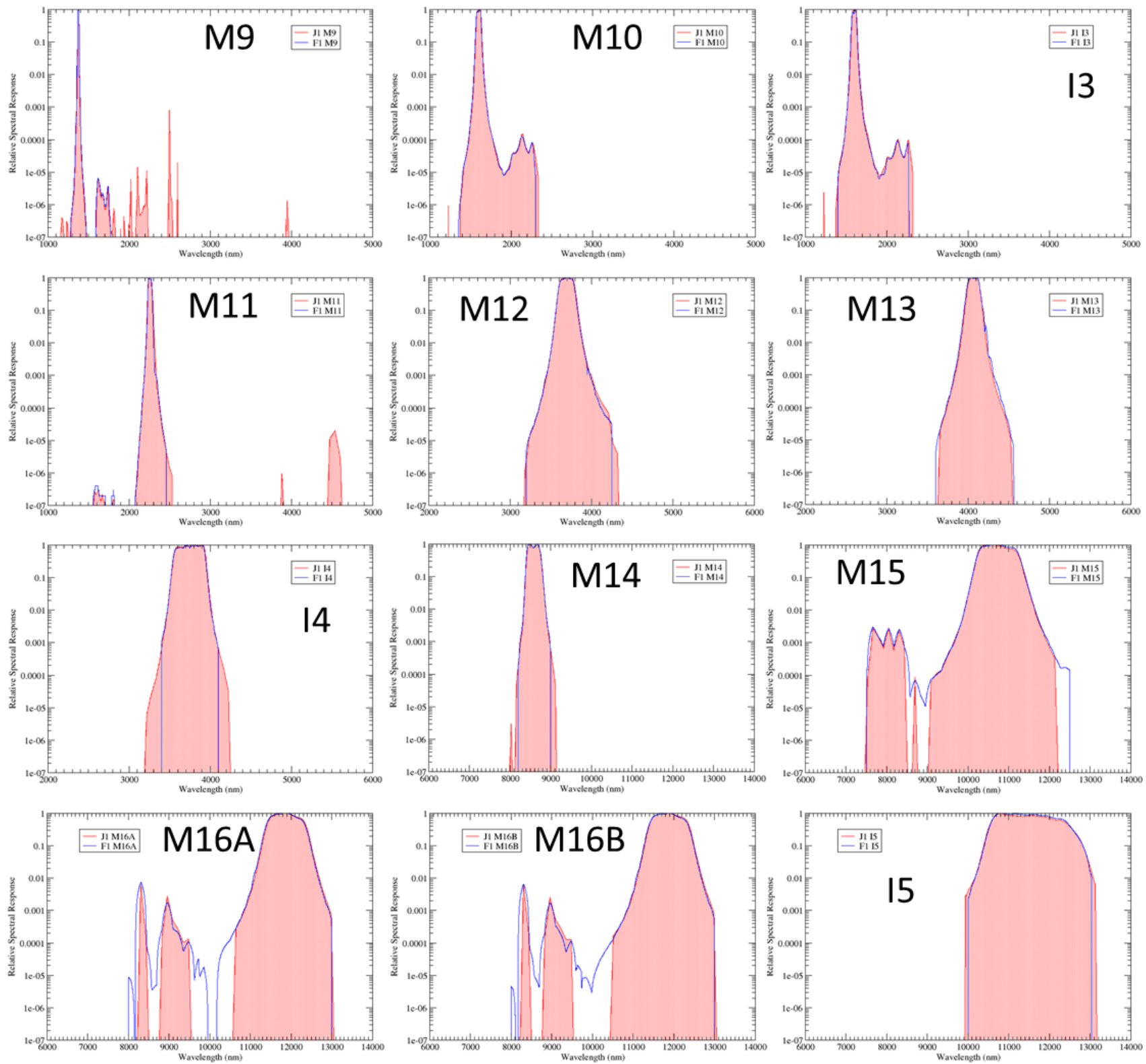

Figure 9 (Cont.). SWIR bands (M9-M11, I3), MWIR bands (M12, M13, I4) and LWIR bands (M14-M16A/B, I5) all show comparable out-of-band response to that of S-NPP. Some elimination of low out-of-band response (<1E-04) in M15 and M16A/B may be a result of data quality differences between J1 and S-NPP measurements. 


\section{VISNIR BAND RSR UNCERTAINTY}

An estimate of wavelength and response uncertainty associated with the T-SIRCUS VisNIR RSR measurements can be based upon the variability of the data collected during the measurements. At each measured wavelength, standard deviation statistics on laser wavelength, source variability and VIIRS signal were collected in real-time or generated in the analysis. The laser wavelength deviation statistic suggests that wavelength uncertainty is less than $0.1 \mathrm{~nm}$ for the vast majority of the measured wavelengths, with isolated occurrences of up to $0.5 \mathrm{~nm}$, primarily in the $500-600 \mathrm{~nm}$ spectral range.

The VisNIR band response is defined by the VIIRS output signal and the T-SIRCUS output radiance during the measurements (see equations 1-3). The response uncertainty was estimated by perturbing the $\mathrm{dn}$ and $\mathrm{L}_{\text {sph }}$ terms of equation 2 using the available SEM and standard deviation statistics, resp., and calculating the root sum square of the two terms. Uncertainties are generally at .001 or lower for bands M1-M7 and between .01 and .001 for the DNB and I1 and I2, reflecting the low gain and thus low signal levels recorded in those bands during the measurements. There are isolated uncertainties up to 0.01 and higher in all $\mathrm{M}$ bands as well in the out-of-band region, though the response levels recorded at these wavelengths remain in family with neighboring wavelengths. The in-band uncertainty is dominated by the uncertainty in the sphere radiance, which is magnified by the high signal levels of the high response zone, whereas the out-of-band region is dominated by the uncertainty in the VIIRS signal, reflecting the low signal levels in the out-of-band region in most bands. As expected, the poorest performance is in the M3 spectral region where the T-SIRCUS LBO OPO laser instability is largest and in the I1 and I2 out-of-band regions where signal levels are lowest.

\section{SUMMARY}

This paper provides an introduction to the J1 VIIRS at-launch band average RSR through the DAWG February 2016 Version 2 (V2) RSR release. The V2 release, which replaces the V1 release of June 2015, contains updates to VisNIR bands DNBLGS, I1, I2, and M1-M7, improving upon the V1 release by characterizing the presence of electronic crosstalk in those bands through the addition of the December 2014 NIST T-SIRCUS RSR measurements to the analysis to form a fused RSR. Band M13 is also updated in V2 by using a forward model to predict and remove the influence of $\mathrm{CO} 2$ present during M13 RSR measurements. For all bands not updated in V2 (DNBMGS, I3-I5, M8-M12, M14-M16A/B), the V2 release contains the RSR that were released in V1.

There are significant spectral position and shape differences between J1 VIIRS RSR and those of S-NPP VIIRS. A redesign of the VisNIR focal plane to reduce the influence of optical crosstalk in $\mathrm{J} 1$ has resulted in these RSR changes. Importantly the RSR changes are robustly measured and documented for the user community and the changes noted for J1 either improve upon or maintain the spectral performance of S-NPP as described by the spectral performance metrics. The V2 release, including the band average RSR, supporting detector RSR, and readme document are available on the password-protected NASA eRoom at https://jpss-erooms.ndc.nasa.gov/eRoom/JPSSInstruments/VIIRSF2 JPSS1/0 38007. The V2 release will be undergoing review for public release before the anticipated launch of $\mathrm{J} 1$.

\section{ACKNOWLEDGEMENTS}

The authors wish to acknowledge the NIST T-SIRCUS team for their efforts to deploy T-SIRCUS to the Raytheon El Segundo facility and make spectral measurements of VIIRS VisNIR bands in December 2014. The authors also acknowledge the efforts of Joel McCorkel and Brendan McAndrew for their role in the measurement effort and valuable comments during the analysis phase of the measurements. The authors also acknowledge the VIIRS instrument team at Raytheon Corp for their technical and logistical support of the T- 
SIRCUS measurement effort. This work has been funded at the University of Wisconsin by NASA Grant NNX13AI99G and by NOAA STAR Grants NA10NES4400013 and NA15NAS4320001.

\section{REFERENCES}

[1] Murphy, R. P., P. E. Ardanuy, F. De Luccia, J. E. Clement, and C. Schueler, "The visible infrared imaging radiometer suite", in Earth Science Satellite Remote Sensing, Vol. 1, pp. 199-223, Springer-Verlag: New York, USA (2006).

[2] Moeller, C., T. Schwarting, J. McIntire, and D. Moyer, "JPSS-1 VIIRS Pre-launch Spectral Characterization and Performance", Proc. SPIE Vol. 9607, 960711S, doi:10.1117/12.2188658, (2015).

[3] Moeller, C., J. McIntire, T. Schwarting, and D. Moyer, "VIIRS F1 Best Relative Spectral Response Characterization by the Government Team", Proc. SPIE Vol. 8153, 81530K, doi:10.1117/12.894552, (2011).

[4] Moeller, C., J. McIntire, T. Schwarting, D. Moyer, and J. Costa "Suomi NPP VIIRS Spectral Characterization: Understanding Multiple Releases", Proc. SPIE Vol. 8510, 85101S, doi:10.1117/12.980437, (2012).

[5] Xiong, X., N. Che, and W. Barnes, "Terra MODIS On-Orbit Spectral Characterization and Performance", IEEE Trans. Geosci. Remote Sens. 44 (8), pp. 2198-2206, (2006).

[6] Brown, S. W., Eppeldauer, G. P., and Lykke, K. R., "Facility for spectral irradiance and radiance responsivity calibrations using uniform sources", Appl. Opt. 45, 8218-8237, (2006).

[7] Barnes, R. A., S. W. Brown, K. R. Lykke, B. Guenther, J. J. Butler, T. Schwarting, K. Turpie, D. Moyer, F. De Luccia, and C. Moeller "Comparison of two methodologies for calibrating satellite instruments in the visible and near-infrared", Appl. Opt. 54, 10376-10396, (2015).

[8] Clough, S. A., M. W. Shephard, E. J. Mlawer, J. S. Delamere, M. J. Iacono, K. Cady-Pereira, S. Boukabara, and P. D. Brown, "Atmospheric radiative transfer modeling: a summary of the AER codes", Short Communication: J. Quant. Spectrosc. Radiat. Transfer, 91, 233-244, (2005).

[9] Lei, N., X. Xiong, and B. Guenther, "Modeling the detector radiometric response gains of the Suomi NPP VIIRS reflective solar bands", IEEE Transactions on Geoscience and Remote Sensing, 53 (3), 1565-1573, doi:10.1109/TGRS.2014.2345481, (2015). 